\title{
Erratum to: Adrenal hypofunction from histoplasmosis: a literature review from 1971 to 2012
}

\author{
R. J. Koene • J. Catanese • G. A. Sarosi
}

Published online: 1 April 2015

(C) Springer-Verlag Berlin Heidelberg 2015

\section{Erratum to: Infection (2013) 41:757-759}

DOI 10.1007/s15010-013-0486-z

We would like to amend our previous submission by clarifying the suspected country of origin, if applicable, to each reported case of adrenal histoplasmosis listed in the supplementary table. Also we would specifically like to correct the erroneous number of cases reported from Belgium, which was inadvertently combined with other cases reported in Europe. The PubMed literature search of "adrenal histoplasmosis" between 1971 and 2012 yielded a total of 230 cases, including 111 reports of adrenal insufficiency (48.3\%). All changes are reflected in the revised index enclosed.

The online version of the original article can be found under doi:10.1007/s15010-013-0486-z.

Electronic supplementary material The online version of this article (doi:10.1007/s15010-015-0767-9) contains supplementary material, which is available to authorized users.

R. J. Koene $(\bowtie) \cdot$ J. Catanese

Department of Medicine, University of Minnesota Medical Center, 420 Delaware St SE, Minneapolis 55455, Minnesota,

USA

e-mail: koene030@umn.edu

G. A. Sarosi

Veterans Affairs Medical Center, Minneapolis, Minnesota, USA 